\title{
Nuclear Cataract Grade 4
}

National Cancer Institute

\section{Source}

National Cancer Institute. Nuclear Cataract Grade 4. NCI Thesaurus. Code C135196.

A stage of nuclear sclerotic cataract marked by severe yellowing and sclerosis of the lens nucleus. (Modified LOCS II) 